\title{
Peer mentorship for adults with spinal cord injury: a static group comparison between mentees and non-mentees' reported coping strategies
}

\author{
Meredith Anne Rocchi ${ }^{1,2} \cdot$ Walter Zelaya ${ }^{3} \cdot$ Shane Norman Sweet $\mathbb{C}^{1,2}$
}

Received: 4 May 2018 / Revised: 17 August 2018 / Accepted: 20 August 2018 / Published online: 25 September 2018

(c) International Spinal Cord Society 2018

\begin{abstract}
Study design Cross-sectional study

Objectives Determine whether participating in peer mentorship is related to differences in reported use of coping strategies (acceptance of injury and fighting spirit) for adults with spinal cord injury.

Setting Quebec, Canada

Methods A static group comparison design was used to retrospectively compare mentees $(n=68)$ and non-mentees $(n=63)$ on their reported coping strategies. Moderation analyses examined differences on coping strategies, while controlling for years since injury (significant covariate).

Results A significant interaction was found between years since injury and peer mentorship for acceptance of injury and fighting spirit, where mentees living with their injury for longer ( 30 years) reported more use of the acceptance of injury and fighting spirit coping strategies.

Conclusion Peer mentorship programs could serve as a means for promoting positive coping strategies for adults with SCI. To better understand the role of peer mentorship, follow-up studies using more rigorous research methodologies such as cohort or randomized controlled trial study designs should be conducted.
\end{abstract}

\section{Introduction}

After a spinal cord injury (SCI), the majority of people will require some life adjustments such as adapting to their homes [1], integrating new self-care activities [2], and, potentially, learning new coping strategies (i.e., cognitive mechanisms to reduce stress) to help manage these changes [3]. The way people interpret their injury and the strategies they use to cope with these interpretations have a significant influence on their adjustment [4] and have been shown to reduce the impact of the injury [5], above and beyond

Meredith Anne Rocchi, Ph.D.

Meredith.rocchi@mail.mcgill.ca

1 Department of Kinesiology and Physical Education, McGill University, Montreal, Canada

2 Center for Interdisciplinary Research in Rehabilitation of Greater Montreal (CRIR), Montreal, Canada

3 Moelle Epinière et Motricité Quebec, Montreal, Canada moderating factors such as functional independence, sex, age, income, and class [6].

In an effort to identify the coping strategies used by adults with SCI, Elfstrom et al. [7] found that adults tended to find ways of approaching stressors by changing themselves, re-evaluating life values, and looking for ways to diminish the gap between their personal capacities and situational demands, which is a strategy they called "acceptance of injury". They also found that others tried to challenge the stressors in their lives by increasing their personal control over their life circumstances and their efforts to behave independently ("fighting spirit"). Finally, some adults with SCI attempted to find external controls for their stressors and relied on others to help close the gap between personal capacities and situational demands ("social reliance"). Fighting spirit and acceptance of injury are considered to be positive coping strategies, whereas social reliance is considered to be a negative strategy [8].

These coping strategies have been linked with positive and negative outcomes among adults with SCI. Specifically, acceptance of injury has been positively associated with increased well-being [8, 9], increased psychological 
adjustment to SCI [10, 11], and reduced anxiety and depression [12]. Similar results were found for fighting spirit, which appears to hold 1-year post-injury [13], as well as across longer time periods [14]. Social reliance, however, has been shown to promote negative outcomes such as increased distress $[9,15]$, decreased life satisfaction and participation [16], and decreased functional independence [13].

Adults with SCI can learn these coping strategies through cognitive-behavioral training [6] or through the support of their social environment [17], which helps them change their perspective and reframe their appraisals [18]. One additional approach that could help promote the use of coping strategies is peer mentorship. Peer mentorship consists of linking trained peer mentors with fellow adults living with SCI. The premise is that peer mentors are individuals who, through their life experiences with SCI, can provide empathetic understanding, practical advice, and emotional support to their mentees $[19,20]$. Peer mentorship programs are often embedded within SCI community organizations, whereby the structure and delivery of these programs can vary from one community organization to another. Emerging research in peer mentoring for adults with SCI suggests that participation in these programs promotes a number of positive outcomes. These outcomes include enhanced wheelchair training skills [21], lower hospitalization rates [22], better self-management skills [23], improved self-efficacy [24], increased life satisfaction [25], and social participation [26, 27]. The role of SCI peer mentorship in assisting with coping has also been suggested in some qualitative studies [20,28]. Currently, SCI peer mentorship programs have been under-utilized as a potential means for promoting positive coping strategies for adults with SCI.

\section{Present study}

The objective of the present study is to determine whether participation in peer mentorship programs is related to an increase in the reported use of positive coping strategies for adults with SCI. Specifically, this study will use a static group comparison design to retrospectively compare adults with SCI who have participated in peer mentorship (mentees) with those who have not (non-mentees) to determine whether there are any differences in their reported coping strategies, while controlling for demographic and SCI characteristics. It is anticipated that participation in peer mentorship will be associated with increased reports of positive coping strategies (acceptance of injury and fighting spirit) and decreased reports of the social reliance strategy for adults with SCI.

\section{Methods}

\section{Participants}

A convenience sample of mentees $(n=68)$ and nonmentees $(n=63)$ who had an SCI were recruited for the present study from a community-based SCI organization in Canada. ${ }^{1}$ The mentees had to have participated in the organization's peer mentorship program, which is a nonstructured individualized program aimed at enhancing independence and quality of life for adults with SCI. Peer mentors and the directors of the organization found that meaningful interactions typically started on the fourth meeting and previous research has shown that the effects of peer mentorship have been shown to persist for 10 years [27]. As such, the organization identified mentees as those who had received at least 4 peer mentorship sessions in the past 5 years, and non-mentees as individuals who received only 1 short introductory session or no sessions with a peer mentor. Contact lists were generated for both groups and the research team recruited mentees and non-mentees by e-mail and telephone between January and September 2015. In order to participate, they had to be above the age of 18 , have an SCI for at least 1 year, and speak either English or French. Participants were excluded if they reported any serious cognitive impairments. Informed consent was provided and participants were offered a $\$ 25$ gift card for their participation. This study consists of a secondary analysis on this sample of adults with SCI that has already been published [27]. The primary outcome of that study was to examine how participation in peer mentorship was related to motivation, well-being, and participation for adults with SCI.

\section{Materials}

Participants completed the following measures through an online and telephone survey.

\section{Demographic profile}

Participants reported their age, sex, relationship status, education, ethnicity, years since injury, injury level, mobility device, ASIA classification, adapted vehicle and home, and access to adapted transport. All variables are reported in Table 1. For the purposes of the present analyses, the education and mobility device variables were recorded into dichotomous variables (Education: secondary education or

\footnotetext{
${ }^{1}$ A priori power analyses suggested that in order to detect a moderatelarge difference in reported coping strategies between the mentees and non-mentees with an alpha of 0.05 and a power of 0.80 , at least 45 adults with SCI were required for both the mentee and non-mentee groups.
} 
Table 1 Participant demographic and spinal cord injury characteristics

\begin{tabular}{|c|c|c|c|c|}
\hline & \multicolumn{2}{|c|}{$\begin{array}{l}\text { Non-mentees } \\
(n=63)\end{array}$} & \multicolumn{2}{|c|}{ Mentees $(n=68)$} \\
\hline & $M(\mathrm{SD})$ & $n(\%)$ & $M(\mathrm{SD})$ & $n(\%)$ \\
\hline \multicolumn{5}{|l|}{ Demographic variables } \\
\hline Age & $\begin{array}{l}53.0 \\
(12.3)\end{array}$ & & $\begin{array}{l}50.00 \\
(13.0)\end{array}$ & \\
\hline \multicolumn{5}{|l|}{ Sex } \\
\hline Male & & $46(73)$ & & $48(72)$ \\
\hline Female & & $17(27)$ & & $19(28)$ \\
\hline \multicolumn{5}{|l|}{ Relationship status } \\
\hline Single & & $37(59)$ & & $33(49)$ \\
\hline Relationship & & $26(41)$ & & $31(51)$ \\
\hline \multicolumn{5}{|l|}{ Ethnicity } \\
\hline Caucasian & & $62(98)$ & & $64(94)$ \\
\hline Other & & $1(2)$ & & $4(6)$ \\
\hline \multicolumn{5}{|l|}{ Education } \\
\hline Secondary or lower & & $29(46)$ & & $23(34)$ \\
\hline $\begin{array}{l}\text { Post-secondary or } \\
\text { higher }\end{array}$ & & $33(52)$ & & $45(66)$ \\
\hline \multicolumn{5}{|c|}{ Spinal cord injury variables } \\
\hline Years since injury & $\begin{array}{l}20.1 \\
(12.8)^{*}\end{array}$ & & $\begin{array}{l}14.1 \\
(13.5)^{*}\end{array}$ & \\
\hline \multicolumn{5}{|l|}{ Injury level } \\
\hline Paraplegia & & $26(41)$ & & $35(51)$ \\
\hline Tetraplegia & & $35(55)$ & & $32(47)$ \\
\hline \multicolumn{5}{|l|}{ ASIA classification } \\
\hline A & & $25(40)$ & & $32(47)$ \\
\hline B & & $4(6)$ & & $4(6)$ \\
\hline $\mathrm{C}$ & & $9(14)$ & & $11(16)$ \\
\hline $\mathrm{D}$ & & $17(27$ & & $10(15)$ \\
\hline $\mathrm{E}$ & & $2(3)$ & & $2(3)$ \\
\hline \multicolumn{5}{|l|}{ Adapted house } \\
\hline Yes & & $54(86)$ & & $55(81)$ \\
\hline No & & $9(14)$ & & $13(19)$ \\
\hline \multicolumn{5}{|l|}{ Mobility device } \\
\hline Powered wheelchair & & $11(17)$ & & $16(24)$ \\
\hline Other & & $52(83)$ & & $51(75)$ \\
\hline \multicolumn{5}{|l|}{ Adapted transport } \\
\hline Available & & $46(73)$ & & $59(87)$ \\
\hline Not available & & $15(24)$ & & $9(13)$ \\
\hline \multicolumn{5}{|l|}{ Adapted vehicle } \\
\hline Yes & & $38(60)$ & & $47(69)$ \\
\hline No & & $25(40)$ & & $21(25)$ \\
\hline
\end{tabular}

Due to missing observations, some percentages do not equal $100 .{ }^{*} p<$ 0.05 . Only years since injury varied significantly between the mentee and non-mentee group $(t(129)=2.59, p<0.05)$. The frequencies (categorical variables) and group means (continuous variables) on all other variables were similar across both the mentee and non-mentees

post-secondary education; mobility device: powered wheelchairs or other mobility devices).

\section{Coping}

Participants completed the Spinal Cord Lesion-Coping Strategies Questionnaire (SCL-CS) [7, 8], which assesses three coping strategies for adults with SCI. The first, acceptance of injury (4 items), measures the extent life values have been re-evaluated following injury. Fighting spirit (5 items) measures efforts to behave independently, and finally, social reliance ( 3 items) measures how adults become dependent on other social beings. Participants respond to each statement using a 4-point Likert scale from 1 (strongly disagree) to 4 (strongly agree). The original validation studies supported that the subscales had acceptable internal reliability (acceptance of injury $\alpha=$ $0.78-0.79$; fighting spirit $\alpha=0.61-0.73$; social reliance $\alpha=0.68-0.72$ ). Mean scores are calculated to represent each coping domain where higher scores indicate greater affirmation of each type of coping strategy. In this sample, the acceptance of injury and fighting spirit subscales demonstrated strong internal reliability ( $\alpha=0.82$ and 0.87 , respectively), whereas the social reliance subscale did not $(\alpha=0.39)$. Closer inspection of the social reliance items suggested that the items may assess different constructs. For example, "You have to believe that other people are able to help you out" focused on beliefs about other people, whereas "I would feel completely helpless without support from others" and "My lesion has taught me that we are all dependent upon others" focused on dependence on others. However, removing that item from the social reliance subscale did not substantially improve the reliability $(\alpha=0.47)$, as such, a composite score was not calculated. Since this subscale could not be used as per the scale guidelines, social reliance was not included in the subsequent study analyses.

\section{Data analysis}

First, we examined the normality and checked for univariate outliers for all study variables. Next, group differences (mentee vs. non-mentee) on all demographic (sex, relationship status, education, age, and ethnicity) and SCI (injury level, ASIA classification, mobility device, adapted house, adapted vehicle, adapted transportation, and years since injury) variables were examined using $t$-tests and chisquare analyses. We also conducted Pearson correlations analyses between the demographic and SCI variables with acceptance of injury and fighting spirit in order to identify any potential covariates.

Because years since injury differs as a function of whether participants received peer mentoring or not (see Results section), moderation analyses were performed to determine whether participation in peer mentorship programs is related to an increase in the reported use of positive coping strategies for adults with spinal cord injury. Moderation was 
analyzed using the PROCESS macro for SPSS (Model 1) [29]. To yield standardized coefficients, all variables were converted to $z$-scores prior to analysis. PROCESS calculates a bias-corrected and accelerated bootstrapped confidence interval $(10,000$ resamples) for the size of the interaction effect (Peer mentorship*years since injury), with significant moderation indicated by a confidence interval that does not contain zero. Change in $R^{2}$ indicated the size of the effect where $0.02,0.13$, and 0.26 were indicative of small, medium, and large effects, respectively [30].

\section{Results}

Means, standard deviations, and correlations between the demographic and SCI variables are listed in Table 1. Data patterns were examined to identify missing observations and revealed that some variables (i.e., sex, social reliance, injury level) were missing between 1 and 3 observations. Given that this represented less than $2 \%$ of overall observations, no modifications were made and a pairwise deletion method was used in the analyses when necessary. Descriptive statistics were calculated for the coping strategies variables (see Table 2). Normality tests showed that acceptance of injury (skewness $=-0.32$, kurtosis $=-0.12$ ) had a normal distribution; but that fighting spirit (skewness $=-1.87$, kurtosis $=4.34$ ) had a non-normal distribution. Fighting spirit was log transformed to improve normality and satisfy test assumptions (revised skewness $=-0.49$, kurtosis $=0.50$ ) and, since the variable was reflected during transformation, it was re-reflected to maintain original directionality and interpretation [31]. No univariate outliers were found.

Next, as reported in Sweet et al. [27], the results of the correlational analyses with the spinal cord injury/demographic variables with the independent variable showed there was a significant difference between non-mentees $(M$ $=20.10, \mathrm{SD}=12.80)$ and mentees $(M=14.10, \mathrm{SD}=$ 13.50 ) for years since injury, $t(117)=2.62, p=0.010$. As such, the main analyses will control for participants' years since injury via a moderation analysis. Finally, we examined all correlations of the spinal cord injury/demographic variables with acceptance of injury and fighting spirit (Table 2). Since years injury was not included in these analyses as it was already identified as having a relationship with the independent variable [32]. Sex and education (women and those with post-secondary education) were significantly and positively associated with fighting spirit and were controlled for in the respective analyses.

A series of analyses were performed in order to test whether peer mentorship impacts coping for adults with spinal cord injury, as assessed by their acceptance of their injury and their fighting spirit. First, the interaction between peer mentorship and years since injury on acceptance of injury was significant with a small to moderate effect size $\left(F_{(5127)}=6.86, \quad p<0.001, r^{2}=0.14 ;\right.$ see Table 3$)$. To interpret the moderation, years since injury was graphed as low ( 1 standard deviation below the mean $\sim 6$ ), medium (the mean 18), and high (1 standard deviation above the mean $\sim 30$ ). There were no reported differences between groups at medium years since injury ( 18 years); however, at low years since injury ( $\sim 6$ years), non-mentees reported slightly greater acceptance of injury than mentees and at high years since injury, mentees reported greater acceptance of injury than non-mentees (See Figure 1a and Table 4). Next, the interaction between peer mentorship and years since injury on fighting spirit, while controlling for sex and education, was significant with a small to moderate effect size $\left(F_{(5123)}=4.96, p<0.001, r^{2}=0.17\right.$; Table 3$)$. A similar pattern emerged in the results where there were no differences between groups at medium years since injury. Nonmentees living with their injury for $\sim 6$ years, however, reported slightly higher fighting spirit and mentees living with spinal cord injury for $\sim 30$ years reported greater fighting spirit (Figure1b and Table 4).

\section{Discussion}

The objective of the present study was to determine whether participation in peer mentorship programs was related to greater self-reported use of positive coping strategies for adults with spinal cord injury. Overall, the results found support for this objective and help provide additional evidence for the positive role peer mentorship plays for adults with spinal cord injury.

We found that there was a positive association between peer mentorship and the coping strategies of acceptance of
Table 2 Descriptive statistics of coping strategies and correlations with demographic and SCI variables

\begin{tabular}{|c|c|c|c|c|c|c|c|c|c|}
\hline \multirow[t]{2}{*}{ Coping } & \multicolumn{2}{|c|}{$\begin{array}{l}\text { Descrip- } \\
\text { tive }\end{array}$} & \multicolumn{7}{|c|}{ Correlations } \\
\hline & $M$ & SD & Age & Sex & Relationship & Education & Level & Mobility & Transport \\
\hline Acceptance of injury & 2.9 & 0.8 & -0.06 & 0.13 & 0.05 & 0.11 & 0.07 & 0.07 & 0.12 \\
\hline Fighting spirit & 3.6 & 0.5 & -0.04 & $0.20 *$ & 0.07 & $0.31 *$ & 0.04 & 0.00 & 0.14 \\
\hline
\end{tabular}

$* p<0.05$ 
Table 3 Moderation results

Table 4 Descriptive statistics and mean comparisons for Fig. 1

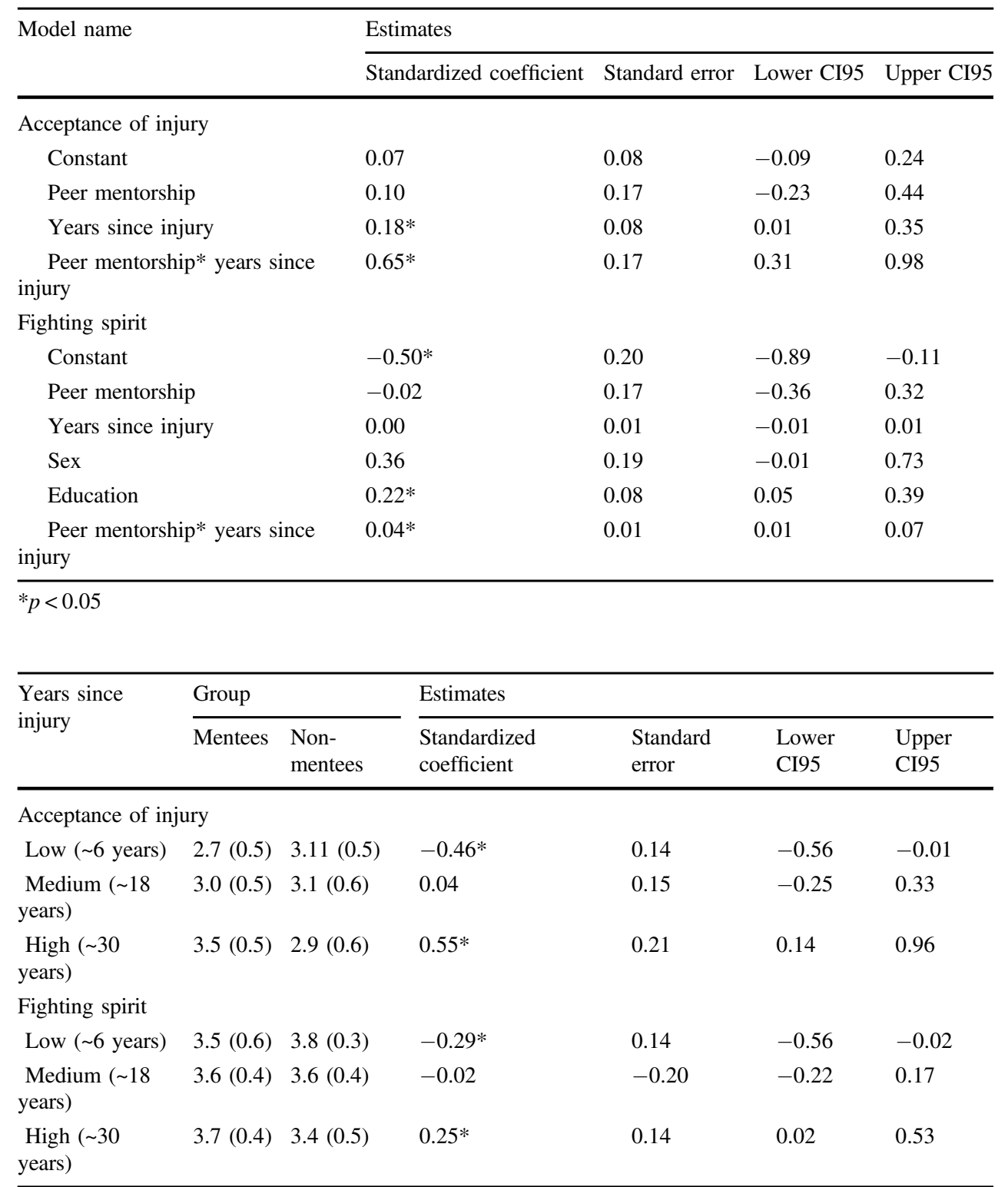

Descriptive statistics are represented as means (standard deviations). ${ }^{*} p<0.05$ injury and fighting spirit (after controlling for participants' education and sex), but only for those who have been living with spinal cord injury for longer. However, the inverse was also found for fewer years since injury. These findings may relate to some studies indicating that the types of coping strategies used for adults with spinal cord injury is always evolving [33], but contrast to other longitudinal research hinting that coping strategies remain relatively stable over time [34]. This moderation effect of years since injury was also seen in the primary outcome of this study [27] that found peer mentorship was positively related to life satisfaction and social participation, but only for those who had been injured for a longer time. In line with these findings, the effects of peer mentorship on coping strategies may be stronger for adults who have been injured longer. It is possible that some factors that are covered through mentorship do not become relevant to the mentee until many years following their injury. It is also plausible that nonmentees who are coping well in the earlier years do not seek peer mentorship, which may be detrimental in the long-term if they do not reach out when in need. There are no published data to compare our findings; thus, more research on peer mentorship and coping strategies is needed before strong conclusions can be made.

We did not examine the relationship between peer mentorship and social reliance as we were unable to establish reliability of that subscale. Since social reliance is considered a negative coping strategy that is associated with outcomes such as increased distress [7], decreased life satisfaction [16], and decreased independence [13], we 
Peer Mentorship and Years Since Injury on Acceptance of Injury

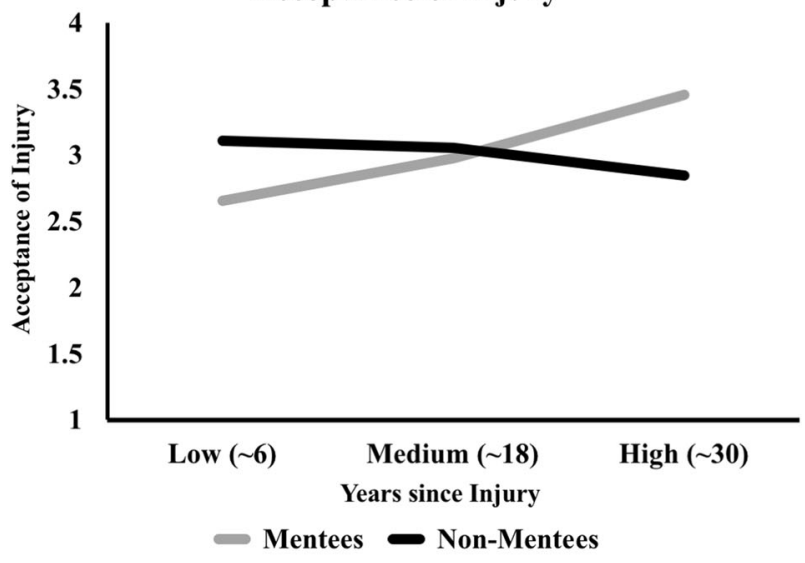

Peer Mentorship and Years Since Injury on Fighting Spirit

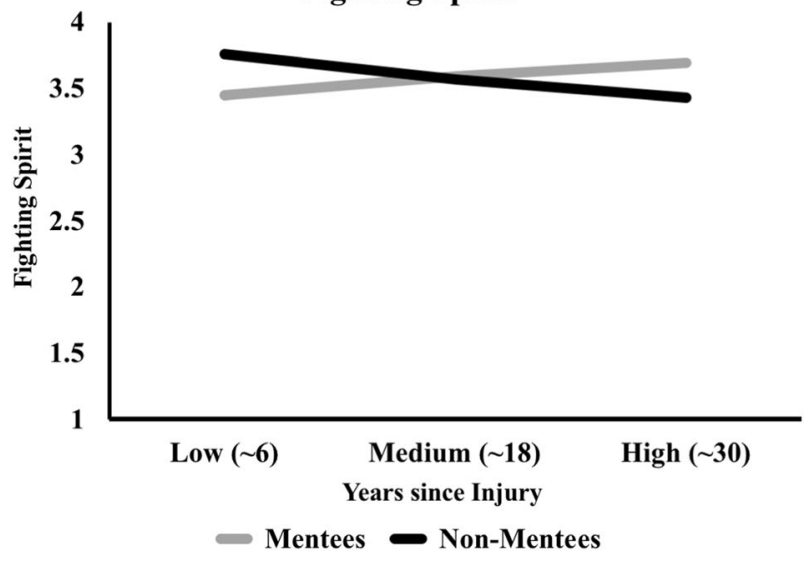

Fig. 1 Interaction between peer mentorship and years since injury on coping strategies. Please note that the Y-Axis (acceptance of injury or fighting spirit) are in their original units of measurement (1-4) in order to ease interpretability

hypothesized that those who participated in peer mentorship would report less social reliance than those who did not. We suspect that the low reliability on this measure was related to the ambiguity of the three items. Specifically, the only item that fully captured the social reliance construct was "I would feel completely helpless without support from others", whereas the other items focused on beliefs that others have the capacity to help when required and we are all dependant on others. It would be important to further examine this construct using a modified or different measure of this construct.

Although not part of our main objectives, the significant relationships between the covariates and coping strategies do provide additional insights. We found that sex and education were significant covariates for the fighting spirit coping strategies, where women and those with a postsecondary education were more likely to endorse this copy strategy. Although existing spinal cord injury research has found mixed results for differences between males and females [7, 8, 35], research from other fields has consistently found that men and women do vary on the types of coping strategies they use [36]. As for the relationship with education, some coping strategies rely on cognitive skills that are enhanced by education [37] and it is possible that those with more education are better equipped to maintain their fighting spirit.

\section{Limitations}

Although this study did provide insight into how participation in peer mentorship programs may be associated with increased use of positive coping strategies for adults with spinal cord injury, the results should be interpreted with caution. To start, this study relied on self-reported data at one single timepoint, thus limiting the comparisons to correlational analyses. Additionally, although the sample consisted of both mentees and non-mentees, this was a community-based static group comparison design with no control or matched comparison group. As such, it is possible that the differences observed between the groups are due to other factors that were not measured as part of this study. Finally, related to the peer mentorship program specifically, we did not collect data related to the frequency of peer mentorship sessions, their satisfaction with these sessions, or whether the sessions occurred individually or in a group format. As a result, the study cannot shed insight into which specific aspects of a peer mentorship program are the most important.

\section{Future directions and practical applications}

The results of this study suggest that peer mentorship programs could serve as a means for promoting positive coping strategies for adults with SCI. To better understand the role of peer mentorship in promoting positive coping strategies, future research should continue to examine the outcomes of participating in peer mentorship for adults with SCI. Specifically, these studies should use stronger methodological approaches (i.e., experimental and longitudinal) in order to fully understand the role peer mentorship plays in impacting the types of strategies used. Additionally, research should look beyond the strategies examined in the present study and explore other types of coping (e.g., reappraisal) [6] and their relationship with peer mentorship. Finally, future research should further explore the role of years since injury and understanding why participating in peer mentorship appears to have a greater influence on those who have been injured longer.

Overall, these findings provide additional support for the positive outcomes associated with participating in peer 
mentorship programs. Since most peer mentorship programs are offered through SCI community-based organizations, these findings may provide additional justification for offering this type of programming and the resources required to run it.

Acknowledgements We would like to acknowledge the contributions of the social integration team of Moelle Epiniere et Motricite Quebec who provided insight across all phases of the study. We also thank Jessica Conti and Keryn Chemtob for helping with recruitment. This study was approved by our institution's research and ethics board and was fully compliant with their guidelines.

Funding This study was supported by the Social Sciences and Humanities Research Council of Canada (Grant no. SSHRC 4302014-00168). Author S. Sweet was a Research Scholar (Junior 1) through the Fonds de recherche du Québec - Santé.

Author contributions SNS was the supervising author on this project and responsible for conceptualizing the project, overseeing data collection, providing input on data analyses, and providing comments on the manuscript. MAR was responsible for conducting the analyses, writing the manuscript, and integrating feedback to the manuscript. WZ was responsible for assisting with the data collection and providing comments on drafts of this manuscript.

\section{Compliance with ethical standards}

Conflict of interest The authors declare that they have no conflict of interest.

\section{References}

1. Chevalier Z, Kennedy P, Sherlock O. Spinal cord injury, coping and psychological adjustment: a literature review. Spinal Cord. 2009;47:778-82.

2. Munce S, Straus S, Fehlings M, Voth J, Nugaeva N, Jang E, et al. Impact of psychological characteristics in self-management in individuals with traumatic spinal cord injury. Spinal Cord. 2016;54:29-33.

3. Song H, Nam K. Coping strategies, physical function, and social adjustment in people with spinal cord injury. Rehabil Nurs. 2010;35:8-15.

4. Galvin L, Godfrey H. The impact of coping on emotional adjustment to spinal cord injury (SCI): review of the literature and application of a stress appraisal and coping formulation. Spinal Cord. 2001;39:615-27.

5. Kennedy P, Lowe R, Grey N. Traumatic spinal cord injury and psychological impact: a cross-sectional analysis of coping strategies. Brit J Clin Psychol. 1995;34:627-39.

6. Kennedy P, Duff J, Evans M, Beedie A. Coping effectiveness training reduces depression and anxiety following traumatic spinal cord injuries. Brit J Clin Psychol. 2003;42:41-52.

7. Elfstrom M, Ryden A, Kreuter M, Persson L, Sullivan M. Linkages between coping and psychological outcome in the spinal cord lesioned: development of SCL-related measures. Spinal Cord. 2002;40:23-29.

8. Elfstrom M, Kennedy P, Lude P, Taylor N. Condition-related coping strategies in persons with spinal cord lesion: a crossnational validation of the spinal cord lesion-related coping strategies questionnaire in four community samples. Spinal Cord. 2007;45:420-8.

9. Elfstrom M, Ryden A, Kreuter M, Taft C, Sullivan M. Relations between coping strategies and health-related quality of life in patients with spinal cord lesion. J Rehabil Med. 2005;37:9-16.

10. Kennedy P, Marsh N, Lowe R, Grey N, Short E, Rogers B. A longitudinal analysis of psychological impact and coping strategies following spinal cord injury. Brit $\mathrm{J}$ Health Psych. 2000;5:157-72.

11. Kennedy P, Nolan M, Smithson E. Psychological adjustment to spinal cord injury in Ireland: quality of life, appraisals and coping. Ir J Psychol. 2011;32:116-29.

12. Bonanno G, Kenney P, Galatzer-Levy I, Lude P, Elfstrom M. Trajectories of resilience, depression, and anxiety following spinal cord injury. Rehabil Psychol. 2012;57:236-47.

13. Kennedy P, Lude P, Elfstrom M, Smithson E. Cognitive appraisals, coping and quality of life outcomes: a multi-centre study of spinal cord injury rehabilitation. Spinal Cord. 2010;48:762-9.

14. Kennedy P, Kilvert A, Hasson L. A 21-year longitudinal analysis of impact, coping, and appraisals following spinal cord injury. Rehabil Psychol. 2016;61:92-101.

15. Elfstrom M, Kreuter M, Ryden A, Persson L, Sullivan M. Effects of coping on psychological outcome when controlling for background variables: a study of traumatically spinal cord lesioned persons. Spinal Cord. 2002;40:408-15.

16. Hansen N, Forchheimer M, Tate D, Luera G. Relationships among community reintegration, coping strategies, and life satisfaction in a sample of persons with spinal cord injury. T Spinal Cord Inj Rehab. 1998;4:56-72.

17. Hampton N. Disability status, perceived health, social support, self-efficacy, and quality of life among people with spinal cord injury in the People's Republic of China. Int J Rehabil Res. 2001;24:69-71.

18. Kennedy P, Evans M, Sandhu N. Psychological adjustment to spinal cord injury: the contribution of coping, hope and cognitive appraisals. Psychol Health Med. 2009;14:17-33.

19. Hernandez B, Hayes E, Balcazar F, Keys C. Responding to the needs of the underserved: a peer-mentor approach. SCI Psysoc Pro. 2001;14:142-9.

20. Veith E, Sherman J, Pellino T, Pellino T, Yasui N. Qualitative analysis of the peer mentoring relationship among individuals with spinal cord injury. Rehabil Psychol. 2006;51:289-98.

21. Best K, Miller W, Huston G, Routhier F, Eng J. Pilot study of a peer-led wheelchair training program to improve self-efficacy using a manual wheelchair: a randomized controlled trial. Arch Phys Med Rehab. 2016;97:37-44.

22. Gassaway J, Jones M, Sweatman W, Hong M, Anziano P, DeVault K. Effects of peer mentoring on self-efficacy and hospital readmission after impatient rehabilitation of individuals with spinal cord injury: a randomized controlled trial. Arch Phys Med Rehab. 2017;98:1526-34

23. Houlihan B, Brody M, Everhart-Skeels S, Pernigotti D, Burnett S, Zazula J, et al. Randomized trial of a peer-led, telephone-based empowerment intervention for persons with chronic spinal cord injury improves health self-management. Arch Phys Med Rehab. 2017;98:1067-76.

24. Ljungberg I, Kroll T, Libin A, Gordon S. Using peer mentoring for people with spinal cord injury to enhance self-efficacy beliefs and prevent medical complications. J Clin Nurs. 2011;20:351-8.

25. Sweet S, Noreau L, Leblond J, Martin Ginis K. Peer support need fulfillment among adults with spinal cord injury: relationships with participation, life satisfaction and individual characteristics. Disabil Rehabil. 2016;38:558-65.

26. Sherman J, Devinney D, Sperling K. Social support and adjustment after spinal cord injury: influence of past peer mentoring experiences and current live-in partner. Rehab Psych. 2004;49:140-9. 
27. Sweet S, Michalovic E, Latimer-Cheung A, Fortier M, Noreau L, Zelaya W. Spinal cord injury peer mentorship: applying selfdetermination theory to explain quality of life and participation. Arch Phys Med Rehab. 2018;99:468-76.

28. Haas B, Price L, Freeman J. Qualitative evaluation of a community peer support service for people with spinal cord injury. Spinal Cord. 2013;51:295-9.

29. Hayes A PROCESS: a versatile computational tool for observed variable mediation, moderation, and conditional process modeling. 2012. http://www.afhayes.com/public/process2012.pdf

30. Cohen J. A power primer. Psych Bull. 1992;112:155-9.

31. Tabachnick B, Fidell L. Experimental designs using ANOVA. Thomson/Brooks/Cole; 2007; Belmont, CA.

32. Field A discovering statistics using IBM SPSS statistics. 2013. Sage. London.
33. Hanson S, Buckelew S, Hewitt J, O'Neal G. The relationship between coping and adjustment after spinal cord injury: a 5-year follow-up study. Rehab Psych. 1993;38:41-52.

34. Pollard C, Kennedy P. A longitudinal analysis of emotional impact, coping strategies and post-traumatic psychological growth following spinal cord injury: a 10-year review. Brit J Health Psych. 2007;12:347-62.

35. Krause J, Broderick L. Outcomes after spinal cord injury: comparisons as a function of gender and race and ethnicity. Arch Phys Med Rehab. 2004;85:355-62.

36. Cracium B. Coping strategies, self-criticism and gender factor in relation to quality of life. Procd Soc Behv. 2013;78:466-70.

37. Cano A, Mayo A, Ventimiglia M. Coping, pain severity, interference, and disability: the potential mediating and moderating roles of race and education. J Pain. 2006;7:469-468. 